\title{
ASSOCIATIONS OF INFECTION PREVENTION AND CONTROL MEASURES WITH COVID-19 CASES AND DEATHS IN NURSING HOMES
}

\author{
V. GUION ${ }^{1}$, L. HE HE $^{1}$ H. BLAIN ${ }^{2}$, H. VILLARS 1,3 , G. DUREL ${ }^{4}$, \\ P. DE SOUTO BARRETO ${ }^{1,3}$, Y. ROLLAND 1,3
}

\begin{abstract}
1. Gerontopole of Toulouse, Institute on Aging, Toulouse University Hospital (CHU Toulouse), Toulouse, France; 2. Department of Geriatrics, Montpellier University Hospital, Montpellier, France; 3. CERPOP Centre d'Epidémiologie et de Recherche en santé des POPulations UPS/INSERM UMR 1295, Toulouse, France; 4. MCoor Association nationale des médecins coordonnateurs et du secteur médico-social, Paris, France. Corresponding author: Vincent GUION, Gerontopole, 20 rue du Pont Saint-Pierre, Cité de la Santé, CHU de Toulouse, 31059 Toulouse - France, Phone: (+33) 561145 664, Fax: (+33) 561145 640, e-mail: vincent.guion@gmail.com
\end{abstract}

\begin{abstract}
Background/Objectives: To identify which infection prevention and control (IPC) precautions in longterm care facilities (LTCF) were associated with reduced COVID-19 incidence and mortality among residents and reduced COVID-19 incidence in health care professionals (HCP). Design: Retrospective data on selfassessment of adherence to 101 IPC measures collected via an online questionnaire sent to 825 nursing homes in France in December 2020. Setting and participants: Medical and administrative staff jointly reported data on IPC measures, characteristics of LTCF, counts of residents' COVID-19 deaths and cases, and counts of HCP cases. Measurements: Random forest models were used to identify the most important IPC measures associated with reduced number of COVID-19 deaths and cases in residents and cases in HCP. The identified variables were then included in linear regression models to estimate the association between levels of adherence to each selected IPC measure and COVID-19 deaths and cases. No data on time of IPC measures implementation were collected. Results: Data from 307 LTCF (37.2\%) were collected, accounting for 22,214 residents. A higher number of COVID-19 deaths in residents was associated with a better adherence to physical distancing in group activity rooms. A better adherence by HCP to physical distancing during their mealtimes and break times was associated with fewer COVID-19 cases among residents and HCP. Other IPC measures were not significantly associated with COVID-19 cases or deaths. Conclusion: Physical distancing between residents was more implemented when LTCF had been confronted with COVID-19 deaths. Physical distancing between HCP was associated with fewer COVID-19 cases in residents and HCP, suggesting it may prevent significantly COVID-19 spreading in LTCF. HCP should particularly adhere to physical distancing measures during their mealtimes and break times. A higher adherence to such preventive measures does not require extra material or human resources and may be easily achievable.
\end{abstract}

Key words: Nursing home, COVID-19, health professional, infection prevention and control measure, random forest model.

\section{Introduction}

The prevalence of COVID-19 is considerably larger in longterm care facilities (LTCF) than in the community (1-4) despite progressive implementation of measures of infection prevention and control (IPC) since first outbreaks (5). In January 2021, the World Health Organization (WHO) issued update advice on IPC precautions to be implemented in LTCF in the context of COVID-19 (6), comprising nine critical IPC policies and procedures, four critical measures to ensure early detection of cases, and five immediate precautions to implement when a resident is identified as a suspected or confirmed case.

The adherence to these IPC measures in the long run by health care professionals (HCP), residents, and their relatives is yet challenging (7-9) since IPC measures can impair residents' and their relatives' psychosocial well-being $(8,10,11)$, and HCP's work satisfaction (12).

Qualitative and quantitative reduction in the implementation of recommended IPC precautions in LTCF may be better accepted by HCP and residents but may in turn be less effective in limiting COVID-19 incidence and mortality.

The objectives of this study were to identify which IPC measures in LTCF were associated with reduced COVID19 incidence and mortality among residents and COVID-19 incidence in HCP.

\section{Methods}

\section{Design, data collection and study population}

All 825 LTCF in the Occitania Region of France were sent a questionnaire to self-assess their adherence to 101 IPC measures. Descriptive LTCF-level data which could be associated with COVID-19 spreading were retrieved, including the number of residents (13-17), ownership (17-20), and presence of a laundry service on-site $(6,21,22)$. No residentlevel data were collected. LTCF administrative and medical staff jointly collected data on number of COVID-19 cases among HCP and residents, and number of COVID-19 deaths over up to four possible outbreaks before December 2020. IPC measures could have been implemented at any time before data collection, either before or after COVID-19 cases were diagnosed in the facility, and no data on time of implementation were collected. 
COVID-19 PREVENTION AND CONTROL IN NURSING HOMES

Table 1

Characteristics of participating long term care facilities $(n=307)$

\begin{tabular}{|c|c|c|c|}
\hline & & $\mathbf{N}(\%)$ & Mean (SD) \\
\hline \multicolumn{2}{|c|}{ Number of beds } & & $72.4(30.8)$ \\
\hline \multicolumn{2}{|c|}{ Laundry service } & $266(86.6)$ & \\
\hline \multirow[t]{3}{*}{ Ownership } & Private for-profit & $57(18.6)$ & \\
\hline & Private not-for-profit & $111(36.2)$ & \\
\hline & Public & $139(45.3)$ & \\
\hline \multicolumn{3}{|c|}{ Number of deaths among residents, per 100 residents $(n=204)$} & $2.9(5.5)$ \\
\hline \multicolumn{3}{|c|}{ Number of cases among residents, per 100 residents $(n=204)$} & $18.3(23.4)$ \\
\hline \multicolumn{3}{|c|}{ Number of cases among staff, per 100 residents $(n=200)$} & $9.0(11.9)$ \\
\hline \multicolumn{2}{|c|}{ COVID-free (no deaths and no cases among staff and residents) } & $78(25.4)$ & \\
\hline
\end{tabular}

\section{Outcome measures}

The main outcome measure was the number of COVID-19 residents' deaths, used as a proportion of the total number of beds in the facility. Secondary outcomes were the number of COVID-19 residents' cases and HCP cases, used as proportions of the total number of beds. Each outcome was calculated as follows:

Outcome $=100 \times$ Number of COVID-19 cases (or deaths) $/$ Number of beds

In France, the number of HCP related to the number of residents, whatever public or private institution, is defined by the regional health agency (RHA) and is globally identical from one LTCF to another.

\section{Covariates}

All explored IPC measures were described in Supplemental Figure 1. For each measure, the four possible answers, defining the level of implementation of the IPC measures in the LTCF, were "never", "insufficiently", "regularly" and "always". In practice, all LTCFs in Occitania were invited by email by the RHA and the investigating Hospital to provide a questionnaire to judge the quality of the preventive measures against COVID19 currently engaged in the institution. The LTCF leadership team (i.e., the medical coordinator, the nurse coordinator, and the principal) had to answer the questions. In order to ensure that the answers given were objective and without fear of criticism or derogatory judgment, the results of the survey were not returned by name to the RHA. The questionnaire was directly fulfilled online and validated by the LTCF's team in a way that no missing data on adherence to IPC measures was possible.

Ownership was defined as public, private non-profit and private for-profit. The size of the LTCF was defined as the number of permanent authorized beds.

\section{Statistical analyses}

Random forest (RF) models were used to analyze the importance of each variable (the 101 IPC measures) in predicting the dependent variables (COVID-19 mortality or incidence in residents and COVID-19 incidence in HCP). A 10-fold cross-validation was used for RF model hypertuning over a tree number from 200 to 2000 and an entry number of independent variables from 3 to 13 . Root mean square error (RMSE) was used for hypertuning evaluation and the hypertuning parameters (i.e., tree number and independent variable entry number) yielding the smallest RMSE value were selected as optimal parameters. With the optimal parameters, a final RF model was built for each dependent variable. The importance of each independent variable in the RF model was evaluated using the percentage increase in mean square error (\%IncMSE), which shows the percentage increase in MSE when corresponding independent variable is permuted. A higher \%IncMSE indicates a greater importance of the variable to predict the outcome.

The most important independent variables were finally included in multivariate linear models to obtain regression coefficients, while keeping the number of observations per degree of freedom of each model over ten. Independent variables were used as categorical, with the category "never" being the reference.

$\mathrm{RF}$ models were performed in $\mathrm{R}$ (version 4.0.3). Regression models were performed in Stata (version 16.1).

\section{Results}

A total of 307 LTCF filled out the questionnaire between November 30th, 2020 and December 16th, 2020, describing IPC precautions and practices applied to 22,214 residents. LTCF's characteristics were presented in Table 1.

The three final regression models were presented in Table 2 .

The ten most important variables as identified by the RF model on the number of residents' deaths were presented 


\section{THE JOURNAL OF NURSING HOME RESEARCH SCIENCESC}

Table 2

Final regression models on number of COVID deaths among residents, and number of COVID cases among residents and among healthcare professionals (HCP)

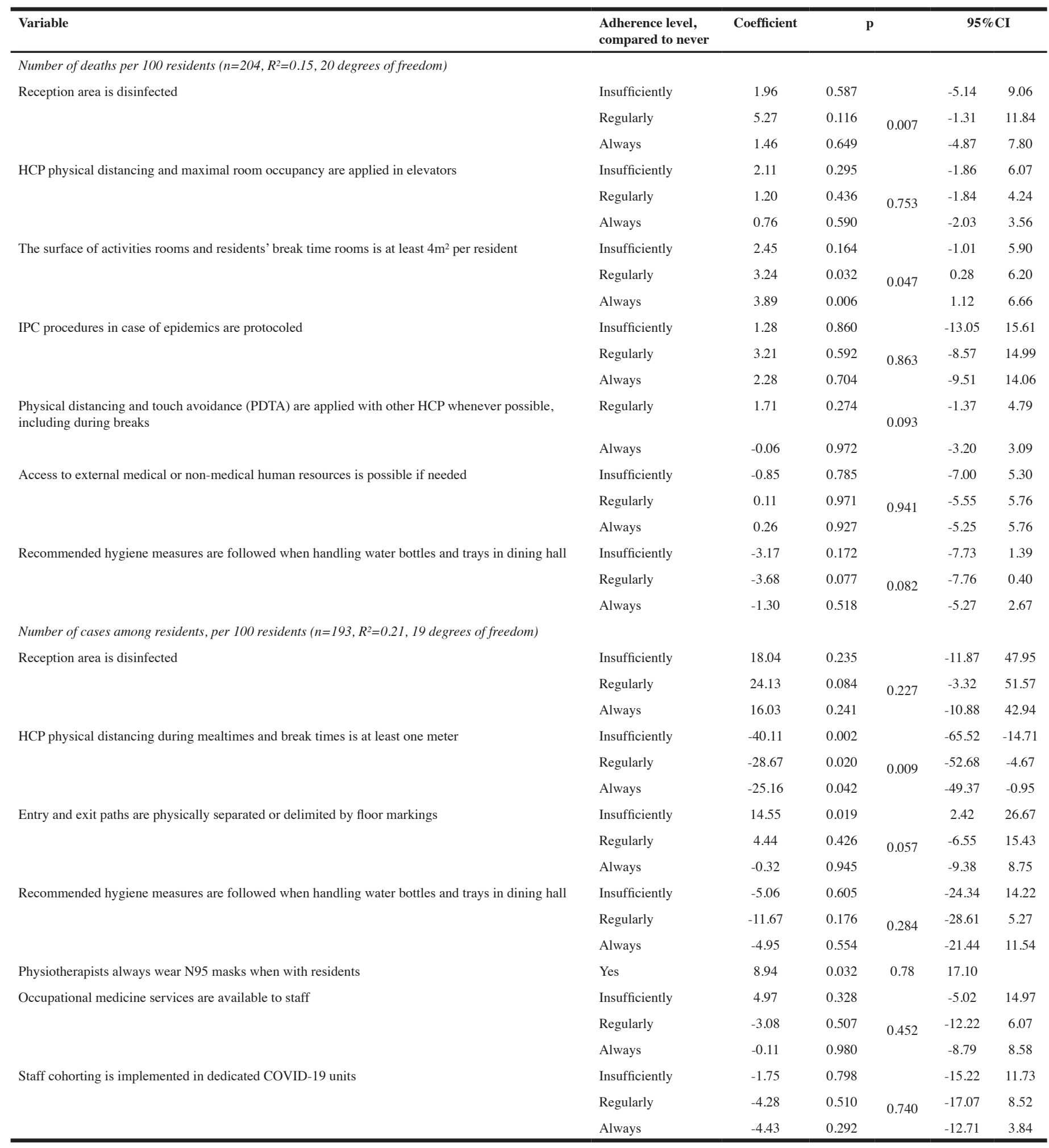


Table 2 (continued)

Final regression models on number of COVID deaths among residents, and number of COVID cases among residents and among healthcare professionals (HCP)

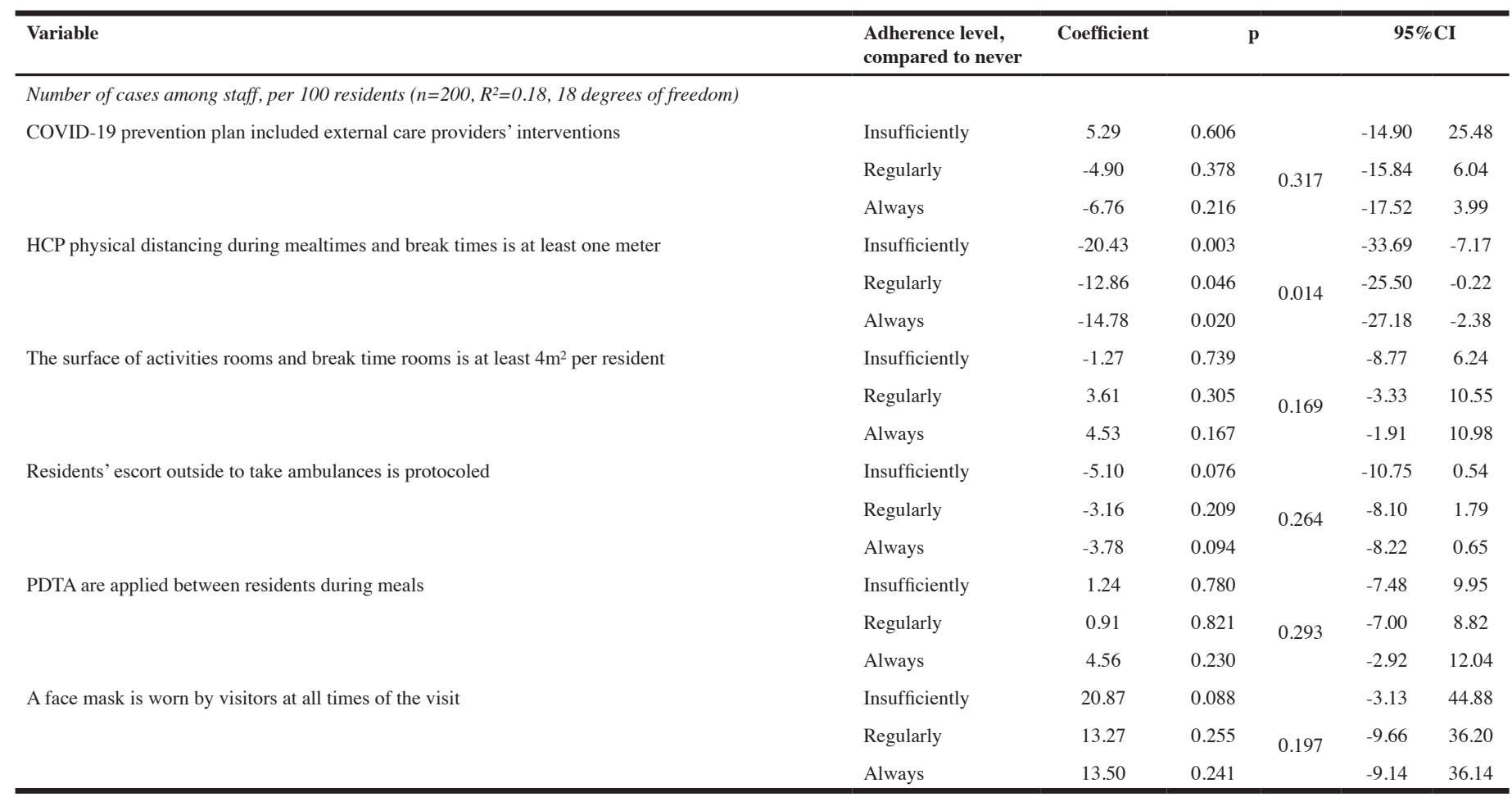

in Supplemental Figure 2. The seven most important IPC measures were used in the regression model on the number of residents' deaths, with \%IncMSE ranging from 4.40 to 7.35 . A higher number of COVID-19 deaths in residents was associated with a better adherence to physical distancing between residents in activity rooms: compared to "never", coefficients for "regularly" and "always" were significantly positive. The number of COVID-19 residents' deaths was also significantly higher in LTCF that implemented reception area disinfection "regularly" rather than "always" (coefficient 3.80 [1.64, 5.96]). None of the five other variables were significantly associated with the number of residents' deaths.

The ten most important variables as identified by the RF model on the number of residents' cases were presented in Supplemental Figure 3. The seven most important IPC measures were used in the regression model on the number of residents' cases, with \%IncMSE ranging from 2.34 to 3.73 . A better adherence by HCP to physical distancing of at least onemeter during their mealtimes and break times was associated with fewer COVID-19 cases among residents: compared to "never", coefficients for "insufficiently", "regularly" and "always" were significantly positive. None of the six other variables were significantly associated with the number of residents' cases.

The ten most important variables as identified by the RF model on the number of HCP cases presented in Supplemental Figure 4. The six most important IPC measures were used in the regression model on the number of HCP cases, with \%IncMSE ranging from 2.27 to 3.37. A better adherence by HCP to physical distancing of at least one-meter during their mealtimes and break times was also associated with fewer COVID-19 cases among HCP: compared to "never", coefficients for "insufficiently", "regularly" and "always" were significantly positive. None of the five other variables were significantly associated with the number of $\mathrm{HCP}$ cases.

\section{Discussion}

This study highlights the importance of physical distancing in preventing the spread of COVID-19 in LTCF, especially among HCP during critical moments of the day at work like mealtimes and break times. WHO has widely recommended physical distancing, including between HCP during their breaks (6), suggesting that it prevents transmission of COVID-19 between HCP. The recommended distance was increased from one to two meters in general population in France after the emergence of new variants of SARS-CoV2 (23). Our results confirm that physical distancing of HCP during their breaks is also associated with the prevention of transmission to residents. The applicability and acceptability of such IPC measures 


\section{THE JOURNAL OF NURSING HOME RESEARCH SCIENCESC}

seem high, as they do not require extra staffing nor expensive material, and could be implemented despite shortages of HCP (24).

The level of adherence to certain IPC measures may increase as the number of cases and deaths increases in the LTCF, demonstrating the adaptation of HCP during an epidemic by strengthening precautions. This was illustrated in our study by the association between a fairly abstract measure (the area of activity rooms being at least $4 \mathrm{~m}^{2}$ per resident) and a higher number of resident deaths. We explain this association by considering that the measure was all the more implemented when the institution had been confronted with deaths related to COVID-19.

The use of random forest models was a novel approach and allowed to investigate a high number of IPC measures and levels of adherence. This method was particularly appropriate as the number of participating LTCF was limited, even though it reached almost $40 \%$ of the target sample.

Yet, this study shows several limitations in its methods. The most important limitation must be the absence of resident-level data, excluding the possibility to consider individual outcomes rather than facility-level outcomes. Individual data may also have brought insight into risk factors for lower adherence of residents to some IPC measures, like dementia that could limit the ability of residents to develop new hand hygiene habits. The declarative nature of LTCF's level of adherence may have introduced a selection bias, with the most performing LTCF being more prone to answer, thus introducing a ceiling effect in adherence levels to IPC measures. Ownership is known to play a role in the spread of COVID-19 in LTCF, especially in private for-profit facilities $(18,20)$, and these were surprisingly under-represented in our sample. This may have introduced an underestimation of COVID-19 number of cases and deaths. The nature of the outcomes (the number of cases and deaths) may have been too sensitive for a declarative method of data collection, even though the WHO proposed self-assessment "to help identify, prioritize and address any gaps in IPC capacity" (6). LTCF may have felt responsible for COVID-19 cases or deaths if IPC measures were not sufficiently implemented. This may have introduced a methodological bias as about one LTCF in three did not report cases or deaths counts and were consequently excluded from analyses.

Physical distancing between HCP members was associated with a lower rate of COVID-19, suggesting this IPC measure might prevent COVID-19 spreading in LTCF. HCP should be particularly precautious during their mealtimes and break times, as a lower adherence to physical distancing measures seem to be associated with a higher number of cases among HCP, and among residents. A higher adherence to such preventive measures seems to be easily achievable in all settings, as it does not rely on extra material or human resources.

Conflict of interest: All authors have completed the ICMJE uniform disclosure form (available on request from the corresponding author) and declare: no support from any organization for the submitted work; no financial relationships with any organizations that might have an interest in the submitted work in the previous three years, no other relationships or activities that could appear to have influenced the submitted work; no spouses, partners, or children have financial relationship that may be relevant to the submitted work; and no nonfinancial interests that may be relevant to the submitted work.

Author Contributions: • Study concept and design: Yves Rolland, Hubert Blain, Gaël Durel, Hélène Villars; • Acquisition of data: Yves Rolland, Hubert Blain, Hélène Villars; - Analysis and interpretation of data: Vincent Guion, Lingxiao He, Hubert Blain, Hélène Villars, Gaël Durel, Philipe de Souto Barreto, Yves Rolland; • Drafting of the manuscript: Vincent Guion; - Critical revision of the manuscript for important intellectual content: Vincent Guion, Lingxiao He, Hubert Blain, Hélène Villars, Gaël Durel, Philipe de Souto Barreto, Yves Rolland. All authors had full access to all of the data in the study and first author can take responsibility for the integrity of the data and the accuracy of the data analysis. The corresponding author attests that all listed authors meet authorship criteria and that no others meeting the criteria have been omitted.

Acknowledgements: We would like to thank Christelle Voisin and Cendrine Blazy (Agence Régionale de Santé- Occitania) for their valuable contribution in the study design and data collection.

Sponsor's role: Funding was supported by the CHU Toulouse.

Impact statement: We certify that this work is novel. This research adds to the literature an insight on infection prevention and control measures associated with COVID19 cases and death in long-term care facilities' residents and healthcare professionals Physical distancing between health care professionals may prevent COVID-19 spreading in long-term care facilities, and professionals should be particularly precautious during their mealtimes and break times.

Ethical standards: Ethics statement: Participants were long term care facilities whose medical and administrative staff reported organizational data. No data was collected at the resident level or at the health care professional level. No consent from participants was therefore required.

\section{References}

1. Ballotari P, Guarda L, Giacomazzi E, Ceruti A, Gatti L, Ricci P. [Excess mortality risk in nursing care homes before and during the COVID-19 outbreak in Mantua and Cremona provinces (Lombardy Region, Northern Italy)]. Epidemiol Prev. 2020;44(56 Suppl 2):282-287. doi:10.19191/EP20.5-6.S2.128

2. Telle KE, Grøsland M, Helgeland J, Håberg SE. Factors associated with hospitalization, invasive mechanical ventilation treatment and death among all confirmed COVID-19 cases in Norway: Prospective cohort study. Scand J Public Health. 2021;49(1):41-47. doi:10.1177/1403494820985172

3. Sepulveda ER, Stall NM, Sinha SK. A Comparison of COVID-19 Mortality Rates Among Long-Term Care Residents in 12 OECD Countries. J Am Med Dir Assoc. 2020;21(11):1572-1574.e3. doi:10.1016/j.jamda.2020.08.039

4. Fisman DN, Bogoch I, Lapointe-Shaw L, McCready J, Tuite AR. Risk Factors Associated With Mortality Among Residents With Coronavirus Disease 2019 (COVID-19) in Long-term Care Facilities in Ontario, Canada. JAMA Netw Open. 2020;3(7):e2015957. doi:10.1001/jamanetworkopen.2020.15957

5. Park SY, Choi G, Lee H, et al. Early Intervention Reduces the Spread of COVID-19 in Long-Term Care Facilities in the Republic of Korea. Osong Public Health Res Perspect. 2020;11(4):259-264. doi:10.24171/j.phrp.2020.11.4.16

6. World Health Organization. Infection prevention and control guidance for longterm care facilities in the context of COVID-19: interim guidance, 8 January 2021. Published online 2021. Accessed February 3, 2021. https://apps.who.int/iris/ handle/10665/338481

7. Telford CT, Bystrom C, Fox T, et al. COVID-19 Infection Prevention and Control Adherence in Long-Term Care Facilities, Atlanta, Georgia. J Am Geriatr Soc. Published online December 28, 2020. doi:10.1111/jgs.17001

8. Verbeek H, Gerritsen DL, Backhaus R, de Boer BS, Koopmans RTCM, Hamers JPH Allowing Visitors Back in the Nursing Home During the COVID-19 Crisis: A Dutch National Study Into First Experiences and Impact on Well-Being. J Am Med Dir Assoc. 2020;21(7):900-904. doi:10.1016/j.jamda.2020.06.020

9. Rolland Y, Lacoste M-H, de Mauleon A, et al. Guidance for the Prevention of the COVID-19 Epidemic in Long-Term Care Facilities: A Short-Term Prospective Study. J Nutr Health Aging. 2020;24(8):812-816. doi:10.1007/s12603-020-1440-2

10. Wammes JD, Kolk MSc D, van den Besselaar Md JH, MacNeil-Vroomen PhD JL, Buurman-van Es Rn BM, van Rijn PhD M. Evaluating Perspectives of Relatives of Nursing Home Residents on the Nursing Home Visiting Restrictions During the COVID-19 Crisis: A Dutch Cross-Sectional Survey Study. J Am Med Dir Assoc. 2020;21(12):1746-1750.e3. doi:10.1016/j.jamda.2020.09.031

11. O'Caoimh R, O'Donovan MR, Monahan MP, et al. Psychosocial Impact of COVID19 Nursing Home Restrictions on Visitors of Residents With Cognitive Impairment: A Cross-Sectional Study as Part of the Engaging Remotely in Care (ERiC) Project. 


\section{COVID-19 PREVENTION AND CONTROL IN NURSING HOMES}

Front Psychiatry. 2020;11:585373. doi:10.3389/fpsyt.2020.585373

12. Leontjevas R, Knippenberg IAH, Smalbrugge M, et al. Challenging behavior of nursing home residents during COVID-19 measures in the Netherlands. Aging Ment Health. Published online December 9, 2020:1-6. doi:10.1080/13607863.2020.185769 5

13. Kosar CM, Rahman M. Early Acceleration of COVID-19 in Areas with Larger Nursing Homes and Certificate of Need Laws. J Gen Intern Med. Published online January 28, 2021:1-8. doi:10.1007/s11606-020-06518-2

14. Zimmerman S, Dumond-Stryker C, Tandan M, et al. Nontraditional Small House Nursing Homes Have Fewer COVID-19 Cases and Deaths. J Am Med Dir Assoc. Published online January 26, 2021. doi:10.1016/j.jamda.2021.01.069

15. Gmehlin CG, Munoz-Price LS. Coronavirus disease 2019 (COVID-19) in long-term care facilities: A review of epidemiology, clinical presentations, and containment interventions. Infect Control Hosp Epidemiol. Published online October 2020:1-6. doi:10.1017/ice.2020.1292

16. Burton JK, Bayne G, Evans C, et al. Evolution and effects of COVID-19 outbreaks in care homes: a population analysis in 189 care homes in one geographica region of the UK. Lancet Healthy Longev. 2020;1(1):e21-e31. doi:10.1016/S26667568(20)30012-X

17. Unruh MA, Yun H, Zhang Y, Braun RT, Jung H-Y. Nursing Home Characteristics Associated With COVID-19 Deaths in Connecticut, New Jersey, and New York. J Am Med Dir Assoc. 2020;21(7):1001-1003. doi:10.1016/j.jamda.2020.06.019
18. McGregor MJ, Harrington C. COVID-19 and long-term care facilities: Does ownership matter? CMAJ Can Med Assoc J. 2020;192(33):E961-E962 . doi:10.1503/ cmaj.201714

19. Braun RT, Yun H, Casalino LP, et al. Comparative Performance of Private EquityOwned US Nursing Homes During the COVID-19 Pandemic. JAMA Netw Open. 2020;3(10):e2026702. doi:10.1001/jamanetworkopen.2020.26702

20. Stall NM, Jones A, Brown KA, Rochon PA, Costa AP. For-profit long-term care homes and the risk of COVID-19 outbreaks and resident deaths. CMAJ Can Med Assoc J J Assoc Medicale Can. 2020;192(33):E946-E955. doi:10.1503/cmaj.201197

21. Shrader CD, Assadzandi S, Pilkerton CS, Ashcraft AM. Responding to a COVID-19 Outbreak at a Long-Term Care Facility. J Appl Gerontol Off J South Gerontol Soc. 2021;40(1):14-17. doi:10.1177/0733464820959163

22. Liu M, Maxwell CJ, Armstrong P, et al. COVID-19 in long-term care homes in Ontario and British Columbia. CMAJ Can Med Assoc J. 2020;192(47):E1540-E1546. doi:10.1503/cmaj.201860

23. HCSP. Covid-19: contrôle de la diffusion des nouveaux variants du virus (complément). Haut Conseil de la Santé Publique; 2021. Accessed March 6, 2021. https://www.hcsp.fr/explore.cgi/avisrapportsdomaine?clefr=974

24. Xu H, Intrator O, Bowblis JR. Shortages of Staff in Nursing Homes During the COVID-19 Pandemic: What are the Driving Factors? J Am Med Dir Assoc. 2020;21(10):1371-1377. doi:10.1016/j.jamda.2020.08.002 